\title{
QUEEN'S
UNIVERSITY
BELFAST
}

\section{Using a generic definition of cachexia in patients with kidney disease receiving haemodialysis: a longitudinal (pilot) study}

McKeaveney, C., Slee, A., Davenport, A., Adamson, G., Farrington, K., Fouque, D., Kalantar-Zadeh, K., Mallett, J., Maxwell, P., Mullan, R., Noble, H., O'Donoghue, D., Porter, S., Seres, D. S., Shields, J., Witham, M., \& Reid, J. (2020). Using a generic definition of cachexia in patients with kidney disease receiving haemodialysis: a longitudinal (pilot) study. Nephrology Dialysis Transplantation. https://doi.org/10.1093/ndt/gfaa174

Published in:

Nephrology Dialysis Transplantation

Document Version:

Peer reviewed version

Queen's University Belfast - Research Portal:

Link to publication record in Queen's University Belfast Research Portal

Publisher rights

Copyright 2020 OUP. This work is made available online in accordance with the publisher's policies. Please refer to any applicable terms of use of the publisher.

\section{General rights}

Copyright for the publications made accessible via the Queen's University Belfast Research Portal is retained by the author(s) and / or other copyright owners and it is a condition of accessing these publications that users recognise and abide by the legal requirements associated with these rights.

Take down policy

The Research Portal is Queen's institutional repository that provides access to Queen's research output. Every effort has been made to ensure that content in the Research Portal does not infringe any person's rights, or applicable UK laws. If you discover content in the Research Portal that you believe breaches copyright or violates any law, please contact openaccess@qub.ac.uk. 
Using a generic definition of cachexia in patients with kidney disease receiving haemodialysis: a longitudinal (pilot) study

Clare McKeaveney ${ }^{1}$

Adrian Slee ${ }^{2}$

Gary Adamson ${ }^{3}$

Andrew Davenport ${ }^{4}$

Ken Farrington ${ }^{5}$

Denis Fouque ${ }^{6}$

Kamyar Kalantar-Zadeh ${ }^{7}$

John Mallett ${ }^{3}$

Alexander P. Maxwell ${ }^{8,9}$

Robert Mullan ${ }^{10}$

Helen Noble ${ }^{1}$

Donal O’Donoghue ${ }^{11}$

Sam Porter ${ }^{12}$

David S. Seres ${ }^{13}$

Joanne Shields ${ }^{9}$

Miles Witham ${ }^{14}$

Joanne Reid ${ }^{1 *}$

${ }^{1}$ School of Nursing and Midwifery, Medical Biology Centre, Queen's University Belfast, Belfast, UK.2Division of Medicine, Faculty of Medical Sciences, University College London, London, UK. ${ }^{3}$ School of Psychology, Ulster University, Coleraine Campus, Londonderry, UK. ${ }^{4}$ UCL Centre for Nephrology, Royal Free Hospital, University College London, London, UK. ${ }^{5}$ Renal Unit, Lister Hospital, East and North Hertfordshire University NHS Trust, Stevenage, UK. ${ }^{6}$ Department of Nephrology, Centre Hospitalier Lyon Sud, University Lyon, CARMEN, Pierre-Benite, France. ${ }^{7}$ Division of Nephrology and Hypertension, University of California Irvine, Orange, California 92868, USA. ${ }^{8}$ Centre for Public Health, Queen's University Belfast, Institute of Clinical Science, Royal Victoria Hospital, Grosvenor Road, Belfast, BT12 6BA, Northern Ireland. ${ }^{9}$ Regional Nephrology Unit, Belfast City Hospital, Belfast Health Social Care Trust, Belfast, BT9 7AB, Northern Ireland. ${ }^{10}$ Department of Nephrology, Antrim Area Hospital, Northern Health Social Care Trust, Antrim, BT41 2RL, Northern Ireland. ${ }^{11}$ School of Medicine, University of Manchester, Manchester, UK. ${ }^{12}$ Department of Social Sciences and Social Work, Bournemouth University, UK. ${ }^{13}$ Department of Medicine, Columbia University Medical Centre / New York Presbyterian Hospital, New York. ${ }^{14}$ NIHR Newcastle Biomedical Research Centre, Newcastle University, Newcastle upon Tyne, UK.

*Corresponding author: Professor Joanne Reid, School of Nursing and Midwifery, Medical Biology Centre, Queen's University Belfast, Belfast, UK; j.reid@qub.ac.uk

Abstract

Background 
Research indicates that cachexia is common among persons with chronic illnesses and is associated with increased morbidity and mortality. However, there continues to be an absence of a uniformed disease specific definition for cachexia in chronic kidney disease (CKD) patient populations.

Objective

The primary objective was to identify cachexia in patients receiving haemodialysis (HD) using Evans et al. generic definition, and then follow up on these patients over 12 months.

Method

This was a longitudinal of 106 adult chronic haemodialysis patients attending two hospital HD units in the United Kingdom. Multiple measures relevant to cachexia including body mass index (BMI), muscle mass (Mid Upper Arm Muscle Circumference; MUAMC), handgrip strength (HGS), fatigue (Functional Assessment of Chronic Illness Therapy; FACIT), appetite (Functional Assessment of Anorexia/Cachexia Therapy; FAACT) and biomarkers (C-reactive protein (CRP); serum albumin; haemoglobin, and Erythropoietin Resistance Index (ERI)) were recorded. Baseline analysis included group differences analysed using independent t-test, dichotomized values using the $X^{2}$ test and prevalence were reported using SPSS 24 . Longitudinal analysis was conducted using repeated measures analysis.

Results

One hundred and six patients (30 female and 76 male) were recruited with a mean age of 67.6 years $(S D=13.18)$ and dialysis vintage of 4.92 years $(S D=6.12)$. At baseline, 17 patients were identified as cachectic, having had reported weight loss (e.g. $>5 \%$ over 6 months) or BMI ( $<20$ $\mathrm{kg} / \mathrm{m}^{2}$ ) and three or more clinical characteristics of cachexia (7). Seventy patients were available for analysis after 12 months ( $n=11$ cachectic vs. $n=59$ not cachectic). FAACT and URR statistically distinguished cachectic patients $(p=.001)$. However, measures of weight, BMI, MUAMC, HGS, CRP, ERI and FACIT tended to be worse in cachectic patients.

Conclusion

Globally, cachexia is a serious but frequently under-recognised problem. This is the first study to apply the defined characteristics of cachexia to a representative sample of patients receiving HD. Further larger studies are required to establish a phenotype of cachexia in advanced CKD.

Keywords: Haemodialysis, Cachexia, Phenotype, Definition, longitudinal analysis

What is already known about this subject:

Globally, cachexia is a serious but frequently under-recognised problem.

It is present in a range of chronic illnesses including cancer, cardiac disease, chronic obstructive pulmonary disease, rheumatoid arthritis and chronic kidney disease. 
It is associated with increased morbidity and mortality including, lower quality of life, increased depression, higher rates of hospitalization and increased risk of death from cardiovascular disease. However, there is limited evidence about the presence of cachexia in end-stage renal disease.

What this study adds:

According to a consensus (generic) definition, cachexia is prevalent in patients with renal disease receiving haemodialysis.

\section{What key message do you want to share with readers?}

This is the first study to apply a consensus definition of cachexia to a population of patients with renal disease and receiving haemodialysis.

This study helps to demonstrate the prevalence of cachexia in end-stage renal disease including the impact on quality of life.

In addition, this study helps to demonstrate the challenges of recruiting patients and retaining individuals within longitudinal research in this patient group.

What impact this may have on practice or policy:

Further research is required to understand if cachexia can be identified as binary (present/absent) or still needs to be considered a process of bodily wasting in renal disease.

Overall, given the impact of cachexia on quality of life of patients with end-stage renal disease and the associated high mortality, it is imperative to develop a robust definition to allow for future feasibility testing of interventions for cachexia currently absence from renal guideline

\section{Introduction}

Cachexia is present in a range of chronic illnesses including chronic kidney disease (CKD) and is associated with increased morbidity and mortality including, lower quality of life, increased depression, higher rates of hospitalization and increased risk of death from cardiovascular disease (1). Several operational definitions exist for cachexia regarding it as the most severe stage of Protein Energy Wasting (PEW; International Society of Renal Nutrition and Metabolism, 2) to a stand-alone disorder (Society of Sarcopenia, Cachexia and Wasting Disorders; 3). PEW and cachexia tend to be used interchangeably in the literature (4) as both disorders have overlapping characteristics. However, research had led to the development of a clinical phenotype for cancer cachexia and other chronic illnesses (5). Prevalence of cachexia ranges from $5 \%$ in rheumatoid arthritis (severe) to $80 \%$ in cancer patients (6). Wasting syndromes are common in renal disease and is reported across all CKD stages, although it is less common in early CKD (5-9\%) compared to advanced CKD stages (20-30\% in stage 4-5). The highest prevalence of cachexia is in end-stage renal disease (ESRD) and particularly those patients on maintenance dialysis (7). Therefore, the ability to accurately assess and monitor cachexia in CKD is an important but potentially underreported aspect of clinical assessment. 
Diagnosing wasting in CKD by estimating measurements of non-oedematous tissue and muscle mass is challenging (8). Advanced CKD is associated with multiple symptoms, particularly those managed by haemodialysis (HD) (9), experience significant and complex changes to their nutritional status and body composition. These alterations to body composition can further confound identifying and diagnosing cachexia (10). Cachexia in CKD (e.g. tissue weight loss) is often masked (e.g. by oedema/fluctuating hydration status). These factors alongside the lack of a disease specific definition for cachexia in CKD helps to explain why it is less commonly recognized in clinical practice (7).

Table 1. Evans et al. generic definition of cachexia (3)

Criteria

Cut-off point

\begin{tabular}{|c|c|c|c|}
\hline \multirow[t]{3}{*}{$\begin{array}{l}\text { Primary } \\
\text { criteria }\end{array}$} & \multicolumn{2}{|c|}{ Weight loss or a low BMI } & $\begin{array}{c}\text { Weight loss } \geq 5 \% \text { over } 12 \text { months or a BMI } \\
<20 \mathrm{~kg} / \mathrm{m}^{2}\end{array}$ \\
\hline & \multicolumn{2}{|c|}{ Muscle strength } & Low handgrip strength (i.e. $<27 \mathrm{~kg}(\mathrm{~m}) /<16 \mathrm{~kg}(\mathrm{f})$ ) \\
\hline & \multicolumn{2}{|c|}{ Fatigue } & Severe fatigue $<30$ \\
\hline AND & \multicolumn{2}{|c|}{ Anorexia } & Poor appetite $<32$ \\
\hline \multirow{4}{*}{$\begin{array}{c}3 \text { of } 5 \text { of the } \\
\text { following } \\
\text { secondary } \\
\text { criteria }\end{array}$} & \multicolumn{2}{|c|}{ Lean tissue depletion } & Low muscle mass (i.e. $<23.8 \mathrm{~cm}(\mathrm{~m}) /<18.4 \mathrm{~cm}(\mathrm{f})$ ) \\
\hline & \multirow[t]{3}{*}{$\begin{array}{c}\text { Abnormal } \\
\text { biochemistry }\end{array}$} & $\begin{array}{l}\text { Increased } \\
\text { inflammation }\end{array}$ & C-reactive protein $(C R P) \geq 5.0 \mathrm{mg} / \mathrm{L}$ \\
\hline & & Anemia & Haemoglobin $\leq 120 \mathrm{~g} / \mathrm{L}$ \\
\hline & & $\begin{array}{l}\text { Low serum } \\
\text { albumin }\end{array}$ & Serum albumin $\leq 32 \mathrm{~g} / \mathrm{L}$ \\
\hline
\end{tabular}

Evans et al. (3) proposed a generic criteria for diagnosing cachexia in chronic illness with appropriate assessment and cut-off points (Table 1 ) that principally requires evidence of unexplained weight loss, low muscle mass and low muscle strength (e.g. by hand grip strength, HGS), as well as abnormal biochemistry. Evidence is needed to establish if this definition is useful and specific to a HD population, as currently there is no standardized phenotype for the identification and assessment of cachexia in ESRD. Therefore, the aim of this study was to 
identify cachexia in patients with ESRD receiving HD using Evans et al. definition and then follow up on these patients for 12 months.

\section{Methods}

We report a longitudinal study with adult HD patients between September 2017 and April 2019, who attended two nephrology units within the United Kingdom. Approximately 310 patients with ESRD receiving HD are cared for between the two nephrology sites. A prospective sample size was calculated. This study sought to and recruited 106 patients which would satisfy a $80 \%$ confidence level. Patients were eligible for inclusion if they had a confirmed diagnosis of ESRD (estimated GFR $<15 \mathrm{~mL} / \mathrm{min} / 1.73 \mathrm{~m}^{2}$ ) and were receiving HD; were able to read and write English and were over 18 years of age (no upper age limit). Patients were excluded those who are Stage 1-4 CKD, Stage 5 CKD who are not receiving haemodialysis, lacking capacity to give consent, under the age of 18 or non- english speaking.

This research collected data (weight, muscle mass, strength, biomarkers, quality of life (KDQoL36) via assessments at point of entry into the study and every two months for one year. A single research assistant carried out all assessments. From these data, a diagnosis of cachexia was made using the definition proposed by Evans et al. definition (3) for cachexia in chronic illness. A minor modification was included and has been validated in the literature (7). This included weight loss $\geq 5 \%$ over 6 months and $\geq 10 \%$ over 12 months allowing for longitudinal assessment (beyond 6 months; 3 ).

\section{Ethics}

Governance approval for the study was obtained from the host institutions and ethics approval from the Office for Research Ethics Committees Northern Ireland prior to the study commencing (Research Ethics Committee reference: 16/NI/0233 and United Kingdom Health Research Authority). All patients provided written informed consent and the study was conducted in accordance with the principles set out in the declaration of Helsinki.

\section{Assessments}

At baseline, demographic data and cachectic status (e.g. weight loss / body mass index (BMI) and recommended clinical characteristics of cachexia) and other information regarding primary renal disease and co-morbidities were recorded. Percentage of weight change of patient's body weight was evaluated at enrollment using medical records. Common biochemical parameters including dialysis efficiency (urea reduction ratio (URR)) and estimated glomerular filtration rate (eGFR) were also recorded. Baseline and longitudinal assessment included twice monthly assessments (+/- one week) of: weight loss of at least $5 \% \geq$ over 6 months or $10 \% \geq$ over 12 months or less; or a BMI $<20 \mathrm{~kg} / \mathrm{m}^{2}$. Additional criteria included lean tissue depletion (using Mid-Upper Arm Muscle Circumference (MUAMC)), reduced handgrip strength, fatigue and abnormal biochemistry; increased inflammatory markers (CRP $\geq$ $5.0 \mathrm{mg} / \mathrm{L}$ ), anaemia (haemoglobin $\leq 120 \mathrm{~g} / \mathrm{L}$ ) and low serum albumin $(\leq 32 \mathrm{~g} / \mathrm{L}$ ). Erythropoietin resistance index (ERI) was also calculated as a surrogate marker for anemia in this patient cohort (weekly erythropoiesis stimulating agent (ESA) dose/ weight $(\mathrm{kg}) \times$ haemoglobin level $(\mathrm{g} / \mathrm{L})$. If the patient died at 6 months their data was excluded from longitudinal analysis due to missing data. 
Bioelectrical Impedance Analysis (BIA)

BIA was used to measure phase angle, using a calibrated dual frequency ( 5 and $50 \mathrm{kHz}$ ). Bodystat 1500 MDD device (Bodystat, Isle of Man, British Isles). A standard protocol was followed with all assessments taken in the post-dialysis period with an aim to control for variation in fluid status. Measurements were taken in the supine position, with electrodes attached on the hand and foot contra-lateral to the side of the arterio-venous fistula, and at constant room temperature. Patients with any implantable electronic devices (such as pacemakers) were excluded as per the manufacturer's guidelines. For those patients that could not complete BIA, a BMI was calculated using the clinical formula: weight $(\mathrm{kg})$ divided by height $^{2}(m)$.

Muscle Mass: Mid-Upper Arm Muscle Circumference (MUAMC)

Mid-arm circumference (MAC) and triceps skinfold (TSF) thickness (TSF in triplicate and the average calculated) were measured in the non-fistula arm using a tape measure and Harpenden skinfold caliper set, respectively. MUAMC $(\mathrm{cm})$ was calculated using the formula: MAMC $(\mathrm{cm})=\operatorname{MAC}(\mathrm{cm})-0.314 \times$ TSF $(\mathrm{mm})$ reference. Suitable cut-point values designated 5th percentile as a suitable cut-point for low MUAMC and used normal values (i.e., $<23.8 \mathrm{~cm}$ for men and $<18.4 \mathrm{~cm}$ for women) $(11,12)$.

Muscle strength: Handgrip strength (HGS)

Muscle function includes a range of measures including power, strength, endurance and fatigability. Handgrip strength: Muscle strength was recorded using a standard protocol (dominant arm seated position with elbow at 90 degrees allowing three attempts) (13) and using a dynamometer (Jamar dynamometer, Patterson, Nottingham, UK). Specific cut-off points were applied based on the European Working Group for Sarcopenia in Older People (EWGSOP) for muscle strength (<27 kg for males and <16 kg for females) (14).

Fatigue: FACIT

Fatigue was recorded using Functional Assessment of Chronic Illness Therapy (FACIT v4, 15). Brown and colleagues (16) reported strong correlations between 'chair-rise' time test and FACIT suggesting it is a reliable measure of physical function. Lower scores of the FACIT subscale for fatigue refers to increased fatigue. The optimal cut-off value for FACIT is $<30$ (17).

Anorexia: FAACT

Appetite was recorded using the Functional Assessment of Anorexia/Cachexia Therapy (FAACT) (18). Lower FAACT subscale for anorexia reflects poor appetite. The optimal cut-off value for FAACT is $<32$ (19).

Analysis

Statistical analysis was performed using SPSS Version 24 (20). Baseline analysis included descriptive results at each time point which are presented as mean \pm standard deviation. Sex specific cut-points were applied to relevant data (HGS, MUAMC). Baseline analysis included group differences analysed using independent t-test. Dichotomized values were compared using the $X^{2}$ test. Longitudinal analysis was conducted using repeated measures analysis. Significance for all analyses was taken at a $p$ value of $<.05$ with Bonferroni correction where appropriate. 
Results

Baseline results

One hundred and six patients (30 female and 76 male) were recruited, and at baseline, 17 patients were identified as having cachexia: 13 patients had $>5 \%$ of weight loss and four had a recorded $\mathrm{BMI}<20 \mathrm{~kg} / \mathrm{m}^{2}$. All 17 patients had three or more clinical characteristics of cachexia (3). Eighty-nine patients were identified as not cachectic.

Table 2. Baseline characteristics

\begin{tabular}{|c|c|c|c|c|}
\hline & All $n=106$ & Cachectic $n=17$ & Not cachectic $n=89$ & Sig. \\
\hline Sex, Female (\%) & $30(28)$ & $7(41)$ & $23(25.8)$ & n.s. \\
\hline Age, yrs (sd) & $67.62+/-13.18$ & $66.71+/-11.44$ & $67.80+/-13.54$ & n.s. \\
\hline$\%>65$ years & $63 \%$ & $64 \%$ & $59 \%$ & - \\
\hline Years on dialysis M (sd) & $4.92+/-6.12$ & $2.78+/-3.04$ & $5.34+/-6.49$ & n.s. \\
\hline $\begin{array}{l}\text { Catheter access } \\
\text { (vs. central line) }\end{array}$ & $84.9 \%$ & $76.5 \%$ & $86.5 \%$ & $\underline{n . s .}$ \\
\hline $\mathrm{CCl}$, score & $6.10+/-2.30$ & $6.65+/-1.73$ & $6.00+/-2.39$ & n.s. \\
\hline Diabetes, \% & $52 \%$ & $24 \%$ & $56 \%$ & $\bar{z}+x+x+1$ \\
\hline Cancer, \% & $25 \%$ & $18 \%$ & $26 \%$ & $=$ \\
\hline $\mathrm{BMI} \mathrm{kg} / \mathrm{m}^{2 *}$ & $28.0(23.0-31.3)$ & $23.0(20.0-29.5)$ & $28.0(24.0-32.0)$ & n.s. \\
\hline Weight $\mathrm{kg} / \mathrm{m}^{2 *}$ & $81.3(65.2-91.2)$ & $62.2(55.1-84.7)$ & $82.6(67.4-93.0)$ & n.s. \\
\hline URR $^{*}$ & $.73(69-.77)$ & $.75(.72-.81)$ & $.73(.68-.77)$ & $<.001$ \\
\hline $\mathrm{eGFR}^{*}$ & $8.2(6.5-6.9)$ & $6.8(5.5-6.8)$ & $8.6(6.9-10.7)$ & n.s. \\
\hline Phase Angle $\mathrm{e}^{\mathrm{a}^{*}}$ & $5.1(4.3-5.7)$ & $5.5(4.8-5.8)$ & $4.9(4.15-5.7)$ & n.s. \\
\hline MUAMC, $m / f^{*}$ & $\begin{array}{c}25.0(22.93-27.5) / \\
24.1(21.0-25.9)\end{array}$ & $\begin{array}{l}22.6(21.6-23.4) / \\
23.2(20.7-29.3)\end{array}$ & $\begin{array}{l}25.6(23.6-28.2) / \\
24.2(21.1-25.6)\end{array}$ & n.s. \\
\hline HGS kg m/f* & $\begin{array}{l}21.8(17.4-27.3) / \\
13.6(10.7-21.0)\end{array}$ & $\begin{array}{l}17.5(11.6-22.5) / \\
13.9(12.9-24.8)\end{array}$ & $\begin{array}{l}22.1(17.8-28.5) / \\
13.3(10.5-18.9)\end{array}$ & n.s. \\
\hline $\mathrm{CRP} \mathrm{mg} / \mathrm{L}^{*}$ & $10.6(3.0-19.6)$ & $17.0(6.8-31.8)$ & $8.0(3.0-17.8)$ & n.s. \\
\hline Serum albumin $\mathrm{g} / \mathrm{L}^{*}$ & $37.5(34.0-40.0)$ & $37.0(34.0-39.5)$ & $38.0(34.5-40.0)$ & n.s. \\
\hline Haemoglobin $\mathrm{g} / \mathrm{L}^{*}$ & $113.0(104.0-119.0)$ & $113.0(104.5-124.0)$ & 113.5 (103.5-119.0) & n.s. \\
\hline $\mathrm{ERI}^{*}$ & $37.9(16.3-82.5)$ & $43.3(31.8-92.4)$ & $31.6(15.1-82.7)$ & n.s. \\
\hline FAACT (ACS) & $37.24+/-7.39$ & $30.53+/-10.48$ & $38.52+/-5.90$ & .001 \\
\hline
\end{tabular}




\begin{tabular}{|c|c|c|c|c|}
\hline FACIT & $32.53+/-12.27$ & $27.00+/-12.21$ & $33.58+/-12.07$ & n.s. \\
\hline $\begin{array}{l}\text { KDQOL-36 } \\
\text { Symptoms/problems list }\end{array}$ & $78.71+/-15.24$ & $68.01+/-17.34$ & $80.76+/-14.00$ & n.s. \\
\hline $\begin{array}{l}\text { KDQOL-36 } \\
\text { Effect of kidney disease }\end{array}$ & $78.36+/-20.44$ & $67.47+/-26.17$ & $80.44+/-18.62$ & n.s. \\
\hline $\begin{array}{l}\text { KDQOL-36 } \\
\text { Burden of kidney disease }\end{array}$ & $44.99+/-29.48$ & $40.80+/-24.72$ & $45.79+/-30.37$ & n.s. \\
\hline KDQOL-36 PCS SF-12 & $37.92+/-10.37$ & $33.29(11.04$ & $38.80+/-49.25$ & n.s. \\
\hline KDQOL-36 MCS SF-12 & $48.25+/-11.91$ & $43.01(13.96$ & $49.25+/-11.29$ & n.s. \\
\hline \multicolumn{5}{|c|}{$\begin{array}{l}\mathrm{N}=\text { number; } \mathrm{M}=\text { Mean }\left(\mathrm{SD}=\text { standard deviation); }{ }^{*}=\text { median }\left(\mathrm{IQR}=\text { Interquartile range); }{ }^{\text {a }} \text { Reported by } \mathrm{n}=87\right.\right. \\
\mathrm{BMI}=\text { Body Mass Index; URR=dialysis UREA clearance; eGFR=estimated glomerular filtration rate; MUAMC=Mic } \\
\text { upper arm muscle circumference; HGS= handgrip strength; CRP=C-reactive protein; ERI= erythropoietir } \\
\text { resistance index; FAACT= Functional Assessment of Anorexia Cancer Therapy; FACIT= Functional Assessmen } \\
\text { of Chronic IIIness Therapy; KDQoL-36=Kidney Disease Quality of Life; PCS SF-12= Physical component score } \\
\text { short form 12; MCS SF-12= Mental Component Score short form 12; CCI=Charlson Comorbidity Index; } \mathrm{m} / \mathrm{f} \\
\text { male/female; Yrs=years. }\end{array}$} \\
\hline
\end{tabular}

Table 2 provides mean (M) baseline scores of all, cachectic and not cachectic patients. Bonferroni correction was applied. Cachexia prevalence in this patient sample was $16 \%(n=17)$. Independent t-test demonstrated that only URR $(p<.001)$ and FAACT $(p=.001)$ were significantly different at baseline between those with cachexia and those without. URR was higher in patients with cachexia than those without cachexia $(M=1.2, S D=1.95$ vs. $M=.71, S D=.10)$. Appetite was poorer in patients with cachexia than those without cachexia $(M=30.53$, $\mathrm{SD}=10.48$ vs. $\mathrm{M}-38.52, \mathrm{SD}=5.90)$.

Longitudinal results

Table 3. Attrition information for all patients (and those identified as cachectic at baseline) after 12 months

\begin{tabular}{|c|c|c|c|c|c|c|c|}
\hline & Total & Died & Tx & $\begin{array}{l}\text { Lost to } \\
\text { follow up }\end{array}$ & WD & HPD & Event Free \\
\hline n (\%) & $\begin{array}{c}106 \\
(100 \%)\end{array}$ & $\begin{array}{c}13 \\
(12 \%)\end{array}$ & $\begin{array}{c}14 \\
(13 \%)\end{array}$ & $\begin{array}{c}1 \\
(1 \%)\end{array}$ & $\begin{array}{c}5 \\
(5 \%)\end{array}$ & $\begin{array}{c}3 \\
(3 \%)\end{array}$ & $\begin{array}{c}70 \\
(66 \%)\end{array}$ \\
\hline $\begin{array}{l}\mathrm{n}=\text { cachectic } \\
\text { at baseline }\end{array}$ & $\begin{array}{c}17 \\
(16 \%)\end{array}$ & $\begin{array}{c}5 \\
(29 \%)\end{array}$ & $\begin{array}{c}0 \\
(0 \%)\end{array}$ & $\begin{array}{c}1 \\
(6 \%)\end{array}$ & $\begin{array}{c}0 \\
(0 \%)\end{array}$ & $\begin{array}{c}0 \\
(0 \%)\end{array}$ & $\begin{array}{c}11 \\
(65 \%)\end{array}$ \\
\hline
\end{tabular}




\begin{tabular}{|c|c|c|c|c|c|c|c|}
\hline $\begin{array}{c}\mathrm{n}=\text { not } \\
\text { cachectic at } \\
\text { baseline }\end{array}$ & 89 & 8 & 14 & 0 & 5 & 3 & 59 \\
$(84 \%)$ & $(9 \%)$ & $(16 \%)$ & $(0 \%)$ & $(6 \%)$ & $(3 \%)$ & $(66 \%)$ \\
\hline
\end{tabular}

$\mathrm{N}=$ number; $\mathrm{Tx}=$ transplant; $\mathrm{WD}=$ withdrawn; $\mathrm{HPD}=$ Home peritoneal dialysis

The majority of patients $(n=70)$ were included at the final assessment point (time 6 ; Table 3 ). Thirty patients identified at baseline as not cachectic were not followed up ( 8 died, 14 transplanted, 5 withdrew and 3 started home peritoneal dialysis). Six patients identified at baseline as cachectic were not followed up ( 5 died, 1 lost to follow up). Longitudinal follow up included 11 patients identified as cachectic at baseline and 59 patients identified as not having cachexia (Appendix 2).

Figure 1. Mean change scores of clinical characteristics of cachexia between baseline and follow up $(n=70)$

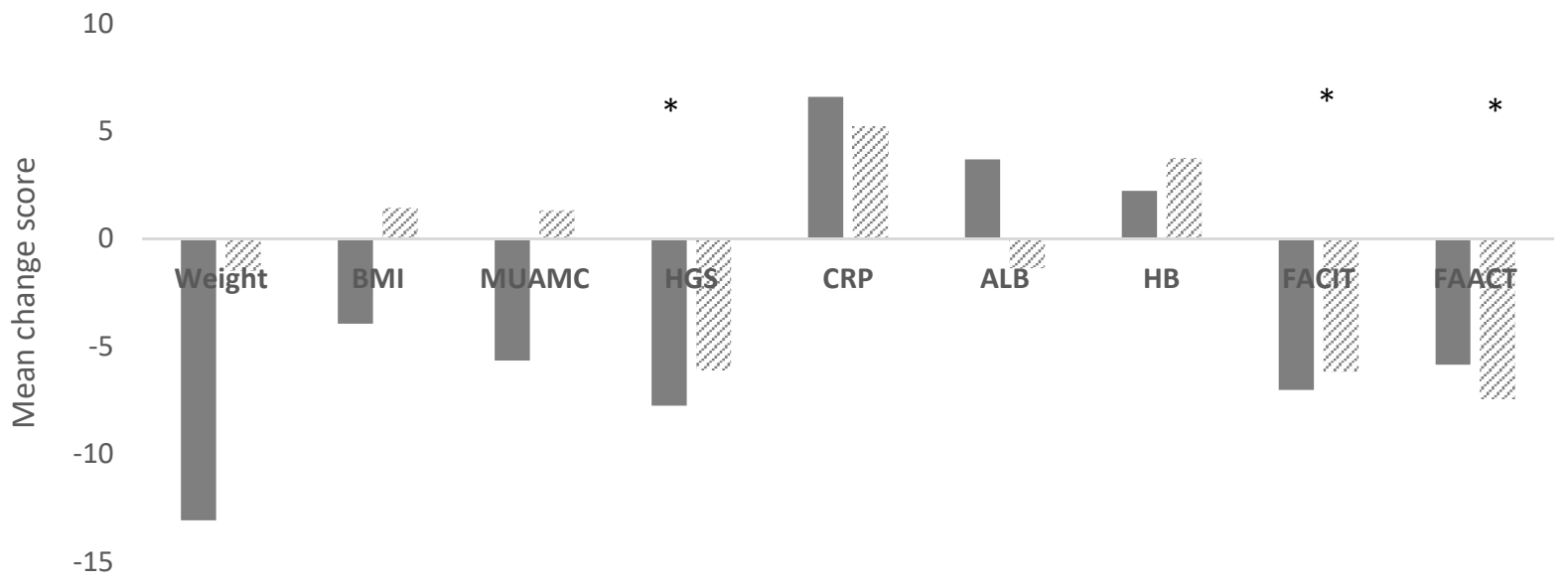

- Cachectic $\succcurlyeq$ Not cachectic

\footnotetext{
*indicates a statistically significance difference, $\mathrm{C}=$ cachectic; $\mathrm{NC}=$ not cachectic; BMI=Body Mass Index; MUAMC= Mid upper arm muscle circumference; HGS= handgrip strength; $\mathrm{CRP}=\mathrm{C}$ reactive protein; $\mathrm{Alb}=$ serum albumin; $\mathrm{Hb}=$ Haemoglobin; $\mathrm{FACIT}=$ Functional Assessment of Chronic Illness Therapy; FAACT= Functional Assessment of Anorexia Cancer Therapy
}

Figure 1 shows significant changes in HGS, FACIT and FAACT after 12 months for the 70 patients who remained in the study. After normality checks a repeated measures analysis was conducted using Greenhouse-Geisser correction for cachectic and not cachectic groups. Cachectic group: showed a significant main effect of time on HGS $(p<.001)$, FACIT $(p<.001)$, and FAACT $(p<.001)$. Post hoc tests with Bonferroni correction revealed significant decreases for FACIT (baseline vs. time 6, $p<.01$ ) and FAACT (baseline vs. time 6, $p=.01$ ). A significant decrease in HGS was reported (baseline vs. time $6, p<.01$ ). Non-cachectic group: showed significant effect of time on HGS $(p<.001)$, FACIT $(p<.001)$ and FAACT $(p<.001)$. FACIT and FAACT declined significantly between baseline and time $6(p=.02, p=.01$ respectively). HGS also significantly decreased (baseline vs. time 6; $p<.05$ ).

\section{Discussion}


This study assessed whether the criteria for cachexia, proposed by Evans et al. (3), could be applied to a representative sample of HD patients at baseline with subsequent follow up every 2 months to a final time point at 12 months. The study evaluated whether cachexia characteristics were present at baseline and to what extent these measurements of cachexia changed over time. The results suggest that cachexia is common in ESRD patients treated with $\mathrm{HD}$, as $16 \%$ of the sample were classified as cachectic at baseline. Reduced appetite and low handgrip strength helped to distinguish between cachectic and non-cachectic patients whereas low muscle mass, fatigue and biomarkers were not. However, despite a lack of significant differences, overall measures of weight, BMI, MUAMC, HGS, CRP, ERI, FAACT and FACIT were worse in those identified as cachectic at baseline. Although anaemia and increased inflammatory markers were common in both groups. Conversely, neither the cachectic or noncachectic groups had significant hypoalbuminaemia. Surprisingly, dialysis adequacy was significantly better in the cachectic group however measurements of URR can vary considerably from treatment to treatment (21). Therefore, alternative measures (e.g. Kt/V) are required.

\section{Cachectic phenotype:}

In absence of recorded weight loss, a BMI of less than $20 \mathrm{~kg} / \mathrm{m}^{2}$ can be used as the primary criterion for cachexia (3). It has been useful in this patient cohort at identifying patients with 'kidney cachexia'. However, BMI requires further investigation. Over $60 \%$ of this patient sample were either overweight or obese which is consistent with other renal studies (22). This may be associated with the higher prevalence of diabetes-related in ESRD (Table 2). In addition, it has been suggested that BMI cut-offs may misrepresent the degree of adverse outcomes in older populations $(23,24)$ and caution should be used when interpreting.

MUAMC provides a measure of muscle mass. After Bonferroni correction, no significant differences between patient groups at baseline were reported. However, clinical cut-offs for low muscle mass were reached indicating muscle catabolism in common in this cohort. Longitudinal assessment highlighted a declining trajectory however this was not statistically significant between baseline and follow up. There is a clear need to routinely monitor muscle mass in CKD, however gold standard assessment measures although more accurate (e.g. DEXA; 8) are difficult to incorporate into routine clinical practice in HD patients. Ventor impedance analysis was not possible due to the small sample size and respective missing data however this technique is useful in CKD patient populations to assess muscle mass independently of the hydration status (25).

Mean scores of muscle strength, measured by HGS were very low in all groups and met clinical cut offs at baseline for cachectic males but not female patients. This may be explained by the small number of females recruited. Longitudinal analysis demonstrated further statistically significant decline in HGS in both groups. According to the EWGSOP recommended criteria, this cohort exhibited clinically low levels of grip strength, an important indicator of sarcopenia and frailty $(14 ;<27 \mathrm{~kg}$ for males; $<16 \mathrm{~kg}$ for females) highlighting potential overlap with other disorders (26). It is also argued that CKD accelerates the aging process which helps to explain why the frailty phenotype is commonly reported in CKD patient populations (27). Such clinical overlap with cachexia requires further investigation in CKD. 
CRP is the most widely agreed biomarker of metabolic abnormality in cancer cachexia (28). Results from the current study also indicate that patients with ESRD receiving HD also have raised CRP levels at multiple time points consistent with chronic inflammation. Evidence suggests that inflammation in patients with advanced CKD is multifactorial and not uncommon (29). Therefore, the cut off value for CRP levels in an ESRD population needs to be revised to a higher level than $5 \mathrm{mg} / \mathrm{L}$.

Levels of serum albumin were not useful in identifying patients with weight loss or cachexia. While albumin concentration is widely used to measure nutritional status this is confounded in patients receiving $\mathrm{HD}$. It is recommended that no single marker should be used to assess nutritional status in renal disease (30). This is because serum albumin is influenced by a range of factors such as fluid balance status, proteinuria and acute inflammation (31). Lower serum albumin levels are also associated with persistent systemic inflammation (32) and may fall further as chronic disease progresses (33). Surprisingly at baseline, albumin was found to be higher in the cachectic group and this trend was observed at the end of the study. Fujiwara and colleagues (34) demonstrated that serum albumin can show substantial intra-day variation, which may help to explain the current results. Longitudinal monitoring should be combined with time of recording as well as comparative assessment.

Similar to serum albumin, haemoglobin did not differ statistically between groups. Clinical practice guidelines suggest that haemoglobin levels in HD patients should be maintained within an optimal range of 100-120g/L with the use of ESAs and intravenous iron. It is therefore not surprising the mean haemoglobin levels of HD patients in this study were below the haemoglobin concentration of $120 \mathrm{~g} / \mathrm{L}$ proposed as a marker for cachexia $(3,35)$. Compared to the trajectory of ERI, a surrogate marker for anemia maintenance, haemoglobin was less useful as a cachexia marker in ESRD (36). As CKD progresses, haemoglobin levels tend to fall necessitating the use of ESAs to increase haemoglobin and reduce the clinical impact of more severe anaemia. Scores of ERI were higher in cachectic patients suggesting such patients require increased dosages of ESAs to remain within the optimal range of $100-120 \mathrm{~g} / \mathrm{L}$.

Patients categorised as cachectic at baseline also reported significantly poorer appetite. Longitudinal analysis also demonstrated appetite significantly decreased for both groups by the end of the study. Prevalence of poor appetite or anorexia is reportedly to range between $25 \%$ to $61 \%$ in ESRD (37) and is associated with increased likelihood of hospitalisation reduced quality of life and higher mortality (38). However, to date, little is known about the direct or indirect impact of anorexia/poor appetite and its relationship with cachexia in ESRD. Prescribed drugs and supplements also interfere with appetite and should be carefully considered when using appetite as a useful predictor of cachexia. Despite this, the FAACT assessments is regarded as a valid tool in HD patients to discriminate anorexia (39).

Participants in the cachectic group also had greater fatigue at baseline, but this was not significantly different at baseline. This is not surprising as fatigue is one of the most frequent reported symptoms and affects $60-97 \%$ of ESRD patients (40). In addition, fatigue increased for both groups by the end of the study. When comparing mean scores for fatigue in cancer patients with cachexia, using the same validated tool as used within this study (FACIT; 15) it is noteworthy that ESRD patients at risk of cachexia demonstrated fatigue similar to or greater than cachectic cancer patients. This helps to contextualise the impact of fatigue on ESRD populations and how this may be severely exacerbated in cachectic ESRD patients (41), an aspect not explored extensively in ESRD. 


\section{Limitations}

The degree of attrition experienced over time in this study is similar to other renal studies (42) and helps to demonstrate the challenges of recruiting patients and retaining individuals within longitudinal research in this patient group. Of note patients did not differ statistically on age or comorbidity levels however this should be considered as confounding the results. It is important to highlight that 52 patients were excluded at the recruitment stage for being "very unwell" (deemed medically unfit to participate by clinical staff; see Appendix 1). Five patients were also withdrawn during the course of the study (Table 3) which may also cause bias. In addition, only one measure of Evans et al. definition (3) statistically distinguished cachectic and non-cachectic patients; a subjective assessment of appetite. Future studies should consider objective assessment of nutritional intake. This study also had a relatively small sample size, with resultant limitations in interpreting the data. Survival analysis was not reported however $29 \%$ of cachectic patients died during the study compared to $9 \%$ of non-cachectic patients. There is a need to examine mortality and associated comorbidities in larger prospective medical data taking account of cachexia. Overall the current study has strengths which include the recruitment and retention of $66 \%$ of the cohort which helps to increase the generalizability of the study.

\section{Conclusion}

Globally, cachexia is a serious but frequently under-recognised problem. This is the first study to apply the known characteristics of cachexia to a representative sample of patients receiving HD. Overall significant differences were limited however measures of muscle strength and reduced appetite are crucial in distinguishing between cachectic and not cachectic patients. Additionally, measures of weight, muscle mass, BMI, CRP, ERI and fatigue were worse in those identified as cachectic at baseline. However, it is important to note this was a pilot study and future studies should aim to increase the sample to reach the sample size to give greater reliability and $95 \% \mathrm{Cl}$ address aforementioned study biases. Further research is required to demonstrate if Evans et al. definition (3) identifies cachexia as binary (present/absent), moving away from a process (1). Given the impact of cachexia on quality of life of patients with ESRD and the associated high mortality, it is imperative to develop a robust definition to allow for future feasibility testing of interventions for cachexia currently absence from renal guidelines.

\section{List of abbreviations}

HD (haemodialysis)

DEXA (dual energy X-ray absorptiometry)

KDQOL-36 (Kidney Disease Quality of Life 36)

eGFR (estimated glomerular filtration rate)

MUAMC (mid upper arm muscle circumference)

ESA (erythropoiesis stimulating agent)

ERI (erythropoiesis resistance index)

MAC (mid-arm circumference) 
TSF (triceps skinfold)

CKD (chronic kidney disease)

ESRD (end-stage renal disease)

BMI (body mass index)

HGS (handgrip strength)

FACIT (Functional Assessment of Chronic Illness Therapy; FACIT)

FAACT (Functional Assessment of Anorexia/Cachexia Therapy)

CRP (C-reactive protein)

EWGSOP (European Working Group for Sarcopenia in Older People)

$\mathrm{Cl}$ (Confidence Interval)

\section{Declarations}

\section{Acknowledgements}

Thanks are given to the patients that participated in this study.

\section{Funding}

This study was funded by the Public Health Agency Northern Ireland [STL/5179/15] and the Northern Ireland Kidney Research Fund.

\section{Availability of data and materials}

The datasets generated and analysed during the current study will be made available in the QUB repository (http://pure.qub.ac.uk/portal/en/datasets/search.html).

\section{Author contributions}

$J R$ is the principal investigator of this study. All authors have assisted in the design of the study and have revised and given approval for the final version. CMcK and JR completed data collection. CM completed data analysis. CMcK and JR completed the initial draft of the manuscript. All authors have read and approved the final manuscript.

\section{Ethical approval}

This study received ethical approval and consent from the Office of Research Ethics Committees Northern Ireland (ORECNI) (REC:16/NI/0233). All participants received a patient information sheet detailing the study and were required to complete a written consent form to participate in the study.

Conflict of interest.

Clare McKeaveney, Adrian Slee, Gary Adamson, Andrew Davenport, Ken Farrington, Denis Fouque, Kamyar Kalantar-Zadeh, John Mallett, Alexander Peter Maxwell, Robert Mullan, Helen Noble, Donal O'Donoghue, Sam Porter, David S. Seres, Joanne Shields, Miles Witham and Joanne Reid declare they have no conflict of interest. 


\section{References}

1. Bonanni, A., Mannucci, I., Verzola, D., Sofia, A., Saffioti, S. et al., 2011. Protein-energy wasting and mortality in chronic kidney disease. Int J Environ Res Public health, 8(5), pp.1631-1654.

2. Fouque, D., Kalantar-Zadeh, K., Kopple, J., Cano, N., Chauveau, P. et al., 2008. A proposed nomenclature and diagnostic criteria for protein-energy wasting in acute and chronic kidney disease. Kidney Int, 73(4), pp.391-398.

3. Evans, W.J., Morley, J.E., Argilés, J., Bales, C., Baracos, V. et al., 2008. Cachexia: a new definition. Am J Clin Nutr, 27(6), pp.793-799.

4. Koppe, L., Fouque, D. and Kalantar-Zadeh, K., 2019. Kidney cachexia or protein-energy wasting in chronic kidney disease: facts and numbers. Journal of cachexia, sarcopenia and muscle.

5. Fearon, K.C., Glass, D.J. and Guttridge, D.C., 2012. Cancer cachexia: mediators, signaling, and metabolic pathways. Cell metabolism, 16(2), pp.153-166.

6. von Haehling, S., Anker, M.S. and Anker, S.D., 2016. Prevalence and clinical impact of cachexia in chronic illness in Europe, USA, and Japan: facts and numbers update 2016. Journal of cachexia, sarcopenia and muscle, 7(5), pp.507-509.

7. Mak RH, Ikizler AT, Kovesdy CP, et al. (2011) Wasting in end stage kidney disease. J Cachexia Sarcopenia Muscle, 2, 9-25.

8. Slee, A., McKeaveney, C., Adamson, G., Davenport, A., Farrington, K. et al., 2019. Estimating the Prevalence of Muscle Wasting, Weakness, and Sarcopenia in Hemodialysis Patients. J Ren Nutr.

9. Reid, J., Noble, H., Slee, A., Davenport, A., Farrington, K. et al., 2016. Distinguishing between Cachexia, sarcopenia and protein energy wasting in end-stage renal disease patients on dialysis. J Palliat Med, 2(2), pp.e11-e13.

10. Uribarri, J., 2018, May. An aspirational diet for dialysis patients: Evidence and theory. In Seminars in dialysis (Vol. 31, No. 3, pp. 236-243).

11. Stosovic, M., Stanojevic, M., Simic-Ogrizovic, S., Jovanovic, D., \& Djukanovic, L. (2011). The predictive value of anthropometric parameters on mortality in haemodialysis patients. Nephrol Dial Transplant. 26(4), pp.1367-1374.

12. Bishop CW, Bowen PE, Ritchey SJ. Norms for nutritional assessment of American adults by upper arm anthropometry. Am J Clin Nutr, 1981 Nov 1;34(11):2530-9.

13. Roberts, H.C., Denison, H.J., Martin, H.J., Patel, H.P., Syddall, H. et al., 2011. A review of the measurement of grip strength in clinical and epidemiological studies: towards a standardised approach. Age ageing, 40(4), pp.423-429.

14. Cruz-Jentoft, A.J., Bahat, G., Bauer, J., Boirie, Y., Bruyère, O., Cederholm, T., Cooper, C., Landi, F., Rolland, Y., Sayer, A.A. and Schneider, S.M., 2018. Sarcopenia: revised European consensus on definition and diagnosis. Age ageing, 48(1), pp.16-31.

15. Cella, D., Eton, D. T., Lai, J. S., Peterman, A. H., \& Merkel, D. E. (2002). Combining anchor and distribution-based methods to derive minimal clinically important differences on the 
Functional Assessment of Cancer Therapy (FACT) anemia and fatigue scales. J Pain Symptom Manage, 24(6), 547-561.

16. Brown DJ, McMillan DC, Milroy R. The correlation between fatigue, physical function, the systemic inflammatory response, and psychological distress in patients with advanced lung cancer. Cancer. 2005 Jan 15;103(2):377-82.

17. Yellen SB, Cella DF, Webster K, Blendowski C, Kaplan E. Measuring fatigue and other anemia-related symptoms with the Functional Assessment of Cancer Therapy (FACT) measurement system. Journal of pain and symptom management. 1997 Feb 1;13(2):63-74.

18. Ribaudo JM, Cella D, Hahn EA, Lloyd SR, Tchekmedyian NS, Von Roenn J, Leslie WT. Revalidation and Shortening of the Functional Assessment of Anorexia/Cachexia Therapy (FAACT) Questionnaire. Qual Life Res. 2000;9(10):1137-46.

19. Turcott, J.G., Oñate-Ocaña, L.F., Soca-Chafre, G., Ramírez-Tirado, L.A., Flores-Estrada, D. et al., 2019. FAACT-anorexia cachexia scale: cutoff value for anorexia diagnosis in advanced nonsmall cell lung cancer patients. Nutr cancer. 71(3), pp.409-417.

20. IBM Corp. Released 2016. IBM SPSS Statistics for Windows, Version 24.0. Armonk, NY: IBM Corp.

21. Daugirdas J. Chronic Hemodialysis Prescription: A Urea Kinetic Approach. In: Daugirdas J, Blake P, Ing T, ed. by. Handbook of Dialysis. 4th ed. Philadelphia, USA: Lippincott Williams \& Wilkins; 2012. p. 146-169.

22. Marcelli, D., Usvyat, L.A., Kotanko, P., Bayh, I., Canaud, B. et al., 2015. Body composition and survival in dialysis patients: results from an international cohort study. Clin J Am SoC Nephrol, 10(7), pp.1192-1200.

23. Batsis, J.A., Mackenzie, T.A., Bartels, S.J., Sahakyan, K.R., Somers, V.K. et al., 2016. Diagnostic accuracy of body mass index to identify obesity in older adults: NHANES 1999-2004. Int J Obes, 40(5), p.761.

24. Madden, A.M. and Smith, S., 2016. Body composition and morphological assessment of nutritional status in adults: a review of anthropometric variables. J Hum Nutr Diet, 29(1), pp.725.

25. Lukaski, H.C., Vega Diaz, N., Talluri, A. and Nescolarde, L., 2019. Classification of hydration in clinical conditions: indirect and direct approaches using bioimpedance. Nutrients, 11(4), p.809.

26. Obi Y, Qader H, Kovesdy CP, Kalantar-Zadeh K. Latest consensus and update on protein energy-wasting in chronic kidney disease. Curr Opin Clin Nutr Metab Care. 2015;18(3):254.

27. Nitta K, Okada K, Yanai M, Takahashi S. Aging and chronic kidney disease. Kidney Blood Press Res. 2013;38(1):109-20.

28. Baracos V, Kazemi-Bajestani SM. Clinical outcomes related to muscle mass in humans with cancer and catabolic illnesses. Int J Biochem Cell Biol, 2013 Oct 1;45(10):2302-8. 
29. Taraz, M., Taraz, S. and Dashti-Khavidaki, S., 2015. Association between depression and inflammatory/anti-inflammatory cytokines in chronic kidney disease and end-stage renal disease patients: A review of literature. Hemodial Int, 19(1), pp.11-22.

30. Fouque, D. and Guebre-Egziabher, F., 2007. An update on nutrition in chronic kidney disease. Int Urol Nephrol, 39(1), pp.239-246.

31. Frimodt-Møller, M., von Scholten, B.J., Reinhard, H., Jacobsen, P.K., Hansen, T.W. et al., 2018. Growth differentiation factor-15 and fibroblast growth factor-23 are associated with mortality in type 2 diabetes-An observational follow-up study. PloS one, 13(4), p.e0196634.

32. Mukai H, Villafuerte H, Qureshi AR, Lindholm B, Stenvinkel P. Serum albumin, inflammation, and nutrition in end-stage renal disease: C-reactive protein is needed for optimal assessment. Semin Dial 2018 Sep (Vol. 31, No. 5, pp. 435-439).

33. Lowrie EG. Chronic dialysis treatment: Clinical outcome and related processes of care. Am J Kidney Dis. 1994;24(2):255-66.

34. Fujiwara, Y., Kobayashi, T., Chayahara, N., Imamura, Y., Toyoda, M., et al., 2014. Metabolomics evaluation of serum markers for cachexia and their intra-day variation in patients with advanced pancreatic cancer. PLoS One, 9(11), p.e113259.

35. Birnie, K., Caskey, F., Ben-Shlomo, Y., Sterne, JA., Gilg, J., et al., Erythropoiesis-stimulating agent dosing, haemoglobin and ferritin levels in UK haemodialysis patients 2005-13. Nephrol Dial Transpl. 2016 Mar 25;32(4):692-8.

36. Jing Z, Wei-jie Y, Nan Z, Yi Z, Ling W. Hemoglobin targets for chronic kidney disease patients with anemia: a systematic review and meta-analysis. PloS One. 2012;7(8):e43655.

37. Murtagh, F.E., Addington-Hall, J. and Higginson, I.J., 2007. The prevalence of symptoms in end-stage renal disease: a systematic review. Advances in chronic kidney disease, 14(1), pp.8299.

38. Kalantar-Zadeh K, Ikizler TA, Block G, Avram MM, Kopple JD. Malnutrition-inflammation complex syndrome in dialysis patients: causes and consequences. Am J Kidney Dis. 2003;42(5):864-81.

39. Molfino A, Kaysen GA, Chertow GM, Doyle J, Delgado C, Dwyer T, Laviano A, Fanelli FR, Johansen KL. Validating appetite assessment tools among patients receiving hemodialysis. J Ren Nutr. 2016 Mar 1;26(2):103-10.

40. Jhamb, M., Liang, K., Yabes, J., Steel, J.L., Dew, M.A. et al., 2013. Prevalence and correlates of fatigue in chronic kidney disease and end-stage renal disease: are sleep disorders a key to understanding fatigue?. Am J Nephrol, 38(6), pp.489-495.

41. Blauwhoff-Buskermolen S, Ruijgrok C, Ostelo RW, de Vet HC, Verheul HM, et al. The assessment of anorexia in patients with cancer: cut-off values for the FAACT-A/CS and the VAS for appetite. Support. Care Cancer. 2016;24(2):661-6.

42. Kovesdy, C.P., 2019. Clinical trials in end-stage renal disease-priorities and challenges. Nephrol Dial Transpl. 34(7), pp.1084-1089. 